Pacific Journal of Mathematics

RELLICH DENSITIES AND AN APPLICATION TO
UNCONDITIONALLY NONOSCILLATORY ELLIPTIC 


\title{
RELLICH DENSITIES AND AN APPLICATION TO UNCONDITIONALLY NONOSCILLATORY ELLIPTIC EQUATIONS
}

\author{
John Piepenbrink
}

\begin{abstract}
Sufficient conditions for embeddings between weighted Sobolev spaces to be compact are derived. These theorems are generalizations of the well known selection principle of Rellich. These results are then applied to the study of the oscillational properties of self-adjoint second order elliptic equations. In addition to reproving some results of Headley and Swanson, new nonoscillation criteria are furnished for these equations.
\end{abstract}

1. Introduction. Let $\Omega$ be a domain, bounded or unbounded, in Euclidean $n$-space $E^{n}, p(x)$ a positive measurable function, $x=$ $\left(x_{1}, \cdots, x_{n}\right)$, and $a(x)$ a symmetric matrix with measurable entries such that the smallest eigenvalue of $a(x)$ for each $x$ in $\Omega$ positive. Define the weighted strong Sobolev spaces $H_{\Omega}(p)$ and $H_{\Omega}(p, a)$ as the closure of the sets of functions $u, C^{1}$ on $\Omega$ for which the integrals

$$
\begin{gathered}
\int_{\Omega} p(x)[u(x)]^{2} d x \\
\int_{\Omega}\left\{p(x)[u(x)]^{2}+\sum a_{i j}(x) u_{i}(x) u_{j}(x)\right\} d x
\end{gathered}
$$

are finite. The closures are taken with respect to norms given by (1.1) and (1.2). The weighted weak Sobolev spaces $W_{\Omega}(p)$ and $W_{\Omega}(p, a)$ consist of functions $u$ with (1.1) or (1.2) respectively being finite. Here $u_{i}(x)$ is the distributional derivative $\partial u / \partial x_{i}$.

We will say that the pair $(p, a)$ has the strong Rellich compactness property if the inclusion map $H_{\Omega}(p, a) \rightarrow H_{\Omega}(p)$ is compact. This means that each sequence in $H_{\Omega}(p, a)$ which is uniformly bounded in its norm has a subsequence which is convergent in the norm for $H_{\Omega}(p)$. The classical Rellich selection principle states that if $\Omega$ is bounded and smooth, then $(1, I)$ has the strong Rellich compactness property where $I$ is the identity matrix. The weak Rellich compactness property is defined analogously with $W_{\Omega}(p), W_{\Omega}(p, a)$ taking the place of $H_{\Omega}(p), H_{\Omega}(p, a)$.

This paper investigates the case where $\Omega=E^{n}, n \geqq 2$. The arguments however apply eqully well to quasi-conical domains, i.e. domains which contain a cone $\{x|x \cdot v \geqq \alpha| x \mid\}$, where $v$ is some unit vector, and $\alpha$ is a positive constant. Theorem 3.1 and 3.2 of $\S 3$ provide sufficient conditions for $(p, a)$ to have either the strong or 
weak Rellich compactness property. Theorem 3.2 is based on a Sobolev type lemma communicated to the author by N. Meyers. A proof of this lemma is included as an appendix. Theorem 3.3 gives a simple condition on $p(x)$ in case $a(x)$ is uniformly definite on $E^{n}$ which ensures that the inclusion map $H_{E^{n}}(p, a) \cap L^{2}\left(E^{n}\right) \rightarrow H_{E^{n}}(p)$ is compact. This weaker result is still sufficient for the application in $\S 5$. In $\S 4$ we give a necessary condition on $p$ when $a$ is uniformly definite. An example shows that the sufficient conditions of Theorems 3.2 and 3.3 are the best of their kind in this case.

In $\S 5$ we apply the preceding results to the determination of the oscillatory properties of the elliptic equation

$$
L u=\sum_{i, j=1}^{n} \frac{\partial}{\partial x_{i}}\left(a_{i j}(x) u_{j}\right)+p(x) u=0 .
$$

We say that (1.3) is nonoscillatory if there is a positive constant $R$ such that for each bounded domain $N$ with smooth boundary exterior to the sphere $\{x|| x \mid \leqq R\}$, the Dirichlet problem

$$
\begin{array}{rll}
L u=0 & \text { in } & N \\
u=0 & \text { on } & \partial N
\end{array}
$$

has nontrivial solution. (1.3) is said to be unconditionally nonoscillatory if for each positive $\lambda,(1.3)$ with $p(x)$ replaced by $\lambda p(x)$ is nonoscillatory.

Theorem 5.1, which asserts that if $(p, a)$ has the Rellich compactness property then (1.3) is unconditionally nonoscillatory, combined with the results of $\S 3$ yields results which differ from the recent. nonoscillation theorems of Swanson and Headley, [1] and [2], in two respects. Our conditions apply directly to the coefficients $a_{i j}(x)$ and $p(x)$, rather than to pointwise majorants. Furthermore our results are not based on the oscillation theory for ordinary differential equations. There is, however, some overlap between the results of [1] and [2] and ours, which will be pointed out later.

2. The case of a bounded domain. Our results for the unbounded domain $E^{n}$ will follow from a process of Cantor diagonalization over compact subdomains. Therefore we will need conditions guaranteeing that $(p, a)$ has a Rellich compctness property for a bounded domain $\Omega$ with smooth boundary $\partial \Omega$. The first result in this direction follows from an imbedding theorem for weighted Sobolev spaces due to Stampachia and Murthy [7], which in turn followed easily from the corresponding result for classical Sobolev spaces, see Sobolev [5]. A special case of the Stampachia-Murthy Theorem is stated as a lemma: 
LEMma 2.1. Let $\Omega$ be a bounded domain in $E^{n}$ with smooth boundary. Suppose $\lambda(x)$ is the smallest eigenvalue of $a(x)$ and $\lambda^{-1}(x)$ and $p^{-1}(x)$ are in $L^{t}(\Omega)$ for some $t \geqq n$. If $1+1 / t<2<n(1+1 / t)$, then the embedding $W_{\Omega}(p, a) \rightarrow L^{q-\varepsilon}(\Omega)$ is compact, where $q$ is defined by

$$
\frac{1}{q}=\frac{1}{2}\left(1+\frac{1}{t}\right)-\frac{1}{n}
$$

From Lemma 2.1 follows our first result.

THEOREM 2.1. Suppose the conditions of Lemma 2.1 hold. If in addition $p(x)$ is in $L^{s}(\Omega)$ where $s=(q-\varepsilon)(q-\varepsilon-2)^{-1}$, then $(p, a)$ has the weak Rellich compactness property on $\Omega$.

By Hölder's inequality

$$
\left[\int_{\Omega} p(x)|u(x)|^{2} d x\right]^{1 / 2} \leqq\left[\left(\int_{\Omega}|u(x)|^{q-\varepsilon} d x\right)^{2 /(q-\varepsilon)}\left(\int_{\Omega}|p(x)|^{s}\right)^{1 / s}\right]^{1 / 2}
$$

or

$$
\left[\int p(x)|u(x)|^{2} d x\right]^{1 / 2} \leqq c\|u\|_{q-\varepsilon},
$$

where $\|u\|_{q-\varepsilon}$ is the norm of $u$ in $L^{q-\varepsilon}(\Omega)$. Thus the imbedding $L^{q-\varepsilon}(\Omega) \rightarrow W_{\Omega}(p)$ is continuous. Since by Lemma 2.1 the imbedding $W_{\Omega}(p, a) \rightarrow L^{q-\varepsilon}(\Omega)$ is compact, and since the composition of a compact map and a continuous map is compact we have the desired conclusion.

A second compactness theorem can be obtained independently of the Sobolev imbedding theorems. Yet despite its apparent simplicity it permits $p(x)$ which are inadmissable in Lemma 2.1. This generality is obtained at the expense of restricting ourselves to the strong spaces.

Theorem 2.2. Let $\Omega$ be bounded and convex and $p(x)$ be nonnegative and measurable. If $p(x)$ is bouded above and has a positive lower bound on some open subset of $\Omega$, then $(p, I)$ has the strong Rellich compactness property.

Proof. Let us assume that $B$ is a bounded subset of $H_{\Omega}(p, I)$. It suffices to assume that $B \subset C^{1}(\Omega)$. Pick $x_{0}$ in $\Omega$ such that $p(x) \geqq p_{0}$ for $\left|x-x_{0}\right| \leqq \varepsilon_{1}$. Thus if $u$ is in $B, \nabla u$ is in $L^{2}(\Omega)$ and $u$ is in $L^{2}\left(S\left(x_{0}, \varepsilon_{1}\right)\right)$, where $S\left(x_{0}, \varepsilon_{1}\right)=\left\{x\left|x-x_{0}\right| \leqq \varepsilon_{1}\right\}$. Now fix $\varepsilon_{0}, 0<\varepsilon_{0}<\varepsilon_{1}$. For any $\varepsilon$ such that $\varepsilon_{0} \leqq \varepsilon \leqq \varepsilon_{1}$ set $u_{\varepsilon}(x)=u\left(x_{0}+\varepsilon\left(x-x_{0}\right) /\left(\left|x-x_{0}\right|\right)\right)$. 
Introduce spherical coordinates $(r, \theta), r=\left|x-x_{0}\right|$ and

$$
\theta=\frac{x-x_{0}}{\left|x-x_{0}\right|}
$$

Then

$$
\begin{aligned}
\int_{\varepsilon_{0}<\left|x-x_{0}\right|<\varepsilon_{1}}|u(x)|^{2} d x & =\int_{\varepsilon_{0}}^{\varepsilon_{1}} r^{r-1} \int_{0}\left|u\left(x_{0}+r \theta\right)\right|^{2} d \theta d r \\
& \geqq \frac{\varepsilon_{1}^{n}-\varepsilon_{0}^{n}}{n} \min _{\varepsilon_{0}<r<\varepsilon_{1}} \int_{\theta}\left|u\left(x_{0}+r \theta\right)\right|^{2} d \theta .
\end{aligned}
$$

So for $u$ in $B$ there is an $\varepsilon, \varepsilon_{0} \leqq \varepsilon \leqq \varepsilon_{1}$ with

$$
\int_{0}\left|u\left(x_{0}+\varepsilon \theta\right)\right|^{2} d \theta \leqq C_{1},
$$

where $C_{1}$ is a constant independent of the particular $u$ in $B$. Now if $R$ is the diameter of $\Omega$, also by (2.2)

$$
\begin{aligned}
\int_{\left|x-x_{0}\right| \geqq \varepsilon}\left|u_{\varepsilon}(x)\right|^{2} d x & \leqq \int_{\varepsilon}^{R} r^{n-1} \int_{\theta}\left|u\left(x_{0}+\varepsilon \theta\right)\right|^{2} d \theta d r \\
& \leqq C_{1} \frac{R^{n}-\varepsilon^{n}}{n}<C_{1} \frac{R^{n}}{n} \equiv C_{2} .
\end{aligned}
$$

So for each $u$ in $B$ there is an $\varepsilon=\varepsilon(u), \varepsilon_{0} \leqq \varepsilon \leqq \varepsilon_{1}$, such that

$$
\int_{\left|x-x_{0}\right| \geqq \varepsilon_{1}}\left|u_{\varepsilon}(x)\right|^{2} d x \leqq C_{2},
$$

and $C_{2}$ is independent of $u$ and $\varepsilon$.

Also if $\left|x-x_{0}\right| \geqq \varepsilon$

$$
u(x)-u\left(x_{0}+\varepsilon \frac{x-x_{0}}{x-x_{0}}\right)=\int_{\varepsilon}^{\left|x-x_{0}\right|} \nabla u\left(x_{0}+s \frac{x-x_{0}}{\left|x-x_{0}\right|}\right) \cdot \frac{x-x_{0}}{\left|x-x_{0}\right|} d s .
$$

Letting $t=s /\left(\left|x-x_{0}\right|\right)$ we see that

$$
\left|u(x)-u_{\varepsilon}(x)\right| \leqq\left|x-x_{0}\right| \int_{\varepsilon /\left(\left|x-x_{0}\right|\right)}^{1}\left|\nabla u\left(x_{0}+t\left(x-x_{0}\right)\right)\right| d t .
$$

Now square both sides and use the Schwartz inequality, integrating over $\left|x-x_{0}\right| \geqq \varepsilon_{1}$ to derive

$$
\int_{\left|x-x_{0}\right| \geqq \varepsilon_{1}}\left|u(x)-u_{\varepsilon}(x)\right|^{2} d x \leqq R^{2} \int_{\left|x-x_{0}\right| \geqq \varepsilon_{1}} \int_{\varepsilon / R}^{1} \mid \nabla u\left(x_{0}+\left.t\left(x-x_{0}\right)\right|^{2} d t d x .\right.
$$

We reverse the integrals on the right and set $y=x_{0}+t\left(x-x_{0}\right)$, obtaining

$$
\int_{\left|x-x_{0}\right| \geqq \varepsilon_{1}}\left|u(x)-u_{\varepsilon}(x)\right|^{2} d x \leqq R^{2} \int_{\varepsilon_{0} / R}^{1} \frac{1}{t^{n}} \int_{\Omega}|\nabla u(y)|^{2} d y d t
$$


or

$$
\int_{\left|x-x_{0}\right| \geqq \varepsilon_{1}}\left|u(x)-u_{\varepsilon}(x)\right|^{2} d x \leqq C_{3} \int_{\Omega}|\nabla u(y)|^{2} d y,
$$

where $C_{3}$ only depends on $R$ and $\varepsilon_{0}$. From (2.3) and (2.4) we finally see that

$$
\begin{aligned}
\int_{\Omega}|u(x)|^{2} d x & =\int_{\left|x-x_{0}\right| \geqq \varepsilon_{1}}|u(x)|^{2} d x+\int_{\left|x-x_{0}\right| \geqq \varepsilon_{1}}|u(x)|^{2} d x \\
& \leqq C_{4}+2 \int_{\left|x-x_{0}\right| \geqq \varepsilon_{1}}\left|u(x)-u_{\varepsilon}(x)\right|^{2} d x+2 \int_{\left|x-x_{0}\right| \geqq \varepsilon}\left|u_{\varepsilon}(x)\right|^{2} d x \\
& \leqq C_{4}+2 C_{3} \int_{\Omega}|\nabla u|^{2} d y+2 C_{2} .
\end{aligned}
$$

Thus there is a $C$ independent of $u$ such that

$$
\int_{\Omega}|u(x)|^{2} d x \leqq C
$$

for all $u$ in $B$.

Now

$$
|u(x+h)-u(x)| \leqq \int_{0}^{|h|}\left|\nabla u\left(x+t \frac{h}{|h|}\right)\right| d t
$$

or

$$
|u(x+h)-u(x)|^{2} \leqq|h| \int_{0}^{|h|}\left|\nabla u\left(x+t \frac{h}{|h|}\right)\right|^{2} d t .
$$

Integrate both sides with respect to $x$ and interchange the order of integration on the right to obtain

$$
\int_{\Omega}|u(x+h)-u(x)|^{2} d x \leqq|h|^{2} \int_{\Omega}|\nabla u(x)|^{2} d x \leqq C^{\prime}|h|^{2} .
$$

By a well known theorem of Bochner, see [6] p. 38, (2.5) and (2.6) imply that $B$ is compact in $L^{2}(\Omega)$ and since $p(x)$ is bounded above, $B$ is compact in $W_{\Omega}(p)$.

We see that the proof of Theorem 2.2 has yielded the following embedding theorem.

THEOREM 2.3. Let $p(x)$ and $\Omega$ be as in Theorem 2.2. Then $H_{\Omega}(p, I)$ is embedded in $L^{2}(\Omega)$ by a compact mapping.

It should be observed that in the applications to differential equations in $\S 5$ only smooth functions are used. Also in many applications $a(x)$ will be uniformly definite and $p(x)$ bounded from below 
and above by positive constants on bounded subdomains $\Omega$ of $E^{n}$. In this case, where Theorem 2.1 applies anyway, the weak Rellich compactness of $(p, a)$ over $\Omega$ follows from the classical Rellich selection principle referred to in $\S 1$.

3. $\Omega=E^{n}$. Throught this and the remaining sections of this paper the hypothesss of Theorem 2.1 or Theorem 2.2 are assumed to hold on each bounded subdomain of $E^{n}$.

Our first compactness result gives conditions on $p(x)$ sufficient for the mapping $H_{E^{n}}(p, I) \rightarrow H_{E^{n}}(p)$ to be compact. So it may be assumed that the hypothesis either of Theorem 2.1 or 2.2 hold on bounded subdomains. Spherical coordinates $(r, \theta), r=|x|, \theta=x /|x|$ are introduced.

THEOREM 3.1. If $p(x)$ satisfies the local conditions of Theorem 2.1 or 2.2 , is continuous and in addition

$$
\lim _{a \rightarrow \infty}\left[\sup _{\theta} \int_{a}^{\infty} r^{n} p(r \theta) d r\right]=0, \text { and } \inf _{\theta} \int_{1}^{a} r^{n-1} p(r \theta) d r>0
$$

then $(p, I)$ has the strong Rellich compactness property.

Proof. The theorem is proved if we can establish the ineqality

$$
\int_{E^{n}} p(x)|u(x)|^{2} d x \leqq \gamma\left[\int_{|x|<a} p(x)|u(x)|^{2} d x+\varepsilon(a) \int_{E^{n}}|\nabla u(x)|^{2} d x\right],
$$

where $\varepsilon(a) \rightarrow 0$ as $a \rightarrow \infty, \varepsilon(a)$ and $\gamma$ are independent of $u$ in $H_{E^{n}}(p, \bar{I})$. For if $\left\{u_{n}\right\}$ is a bounded sequence in $H_{E^{n}}(p, I)$, by the results of the preceding section and Cantor diagonalization we can select a subsequence $\left\{u_{n_{k}}\right\}$ which is Cauchy in $H_{\Omega}(p)$ for each bounded subdomain $\Omega$ of $E^{n}$. But then (3.2) would show that $\left\{u_{u_{k}}\right\}$ was Cauchy in $H_{E^{n}}(\Omega)$, and hence convergent. This would establish the desired conclusion.

Thus we need to prove (3.2) for $u$ in $H_{E^{n}}(p, I)$. Without loss of generality assume that $u$ is in $C^{1}\left(E^{n}\right)$. Fix $\theta$ and let $1 \leqq s<t$. Then

$$
\begin{gathered}
u(t \theta)=u(s \theta)+\int_{s}^{t} \nabla u(s \theta+(\tau-s) \theta) \cdot \theta d \tau \\
u^{2}(t \theta) \leqq 2 u^{2}(s \theta)+2(t-s) \int_{1}^{\infty}|\nabla u(\tau \theta)|^{2} \tau^{n-1} d \tau .
\end{gathered}
$$

Multiply both sides of the inequality by $s^{n-1} p(s \theta)$ and integrate with respect to $s$ from $s=1$ to $s=a$, then divide by $\int_{1}^{a} s^{n-1} p(s \theta) d s$ to derive 


$$
\begin{aligned}
u^{2}(t \theta) \leqq & \frac{2}{\int_{1}^{a} s^{n-1} p(s \theta) d s}\left[\int_{1}^{a} s^{n-1} p(s \theta)|u(s \theta)|^{2} d s+\int_{1}^{a}(t-s) s^{n-1} p(s \theta) d s\right. \\
& \left.\times \int_{1}^{\infty}|\nabla u(\tau \theta)|^{2} \tau^{n-1} d \tau\right] .
\end{aligned}
$$

Now multiply both sides by $t^{n-1} p(t \theta)$ and integrate with respect to $t$ from $t=a$ to $t=\infty$, to find that

$$
\begin{aligned}
\int_{a}^{\infty} t^{n-1} p(t \theta)|u(t \theta)|^{2} d t \leqq & \frac{2 \int_{0}^{a} s^{n-1} p(s \theta)|u(s \theta)|^{2} d s}{\int_{1}^{a} s^{n-1} p(s \theta) d s} \int_{a}^{\infty} t^{n-1} p(t \theta) d t \\
& +\frac{2 \int_{a}^{\infty} \int_{0}^{a} t^{n-1}(t-s) s^{n-1} p(t \theta) p(s \theta) d s d t}{\int_{1}^{a} s^{n-1} p(s \theta) d s} \\
& \times \int_{0}^{\infty}|\nabla u(\tau \theta)|^{2} \tau^{n-1} d \tau .
\end{aligned}
$$

By reversing the order of integrations

$$
\begin{aligned}
\int_{a}^{\infty} \int_{0}^{a} t^{n-1}(t-s) s^{n-1} p(t \theta) p(s \theta) d s d t= & {\left[\int_{0}^{a} s^{n-1} p(s \theta) d s\right]\left[\int_{a}^{\infty} t^{n} p(t \theta) d t\right] } \\
& -\left[\int_{a}^{\infty} t^{n-1} p(t \theta) d t\right]\left[\int_{0}^{a} s^{n} p(s \theta) d s\right] .
\end{aligned}
$$

Thus condition (3.1) shows that if $\varepsilon(a)$ is defined by

$$
\varepsilon(\alpha)=\sup _{0} \int_{a}^{\infty} \int_{0}^{a} t^{n-1}(t-s) s^{n-1} p(t \theta) p(s \theta) d s d t,
$$

then

$$
\varepsilon(a) \longrightarrow 0 \text { as } a \longrightarrow \infty \text {. }
$$

Define

$$
\gamma \geqq 2\left(\inf _{0} \int_{1}^{a} s^{n-1} p(s \theta) d \theta\right)^{-1} \quad \text { for all } a \geqq A \text {. }
$$

Then (3.3) implies

$$
\begin{aligned}
\int_{a}^{\infty} t^{n-1} p(t \theta)|u(t \theta)|^{2} d t \leqq & \gamma\left[\int_{0}^{a} s^{n-1} p(s \theta)|u(s \theta)|^{2} d s\right. \\
& \left.+\varepsilon(a) \int_{0}^{\infty}|\nabla u(\tau \theta)|^{2} \tau^{n-1} d \tau\right] .
\end{aligned}
$$

Now if this is integrated over all $\theta$, (3.2) results.

It is clear from the proof that the same conclusion follows if the condition 


$$
\inf _{\theta} \int_{1}^{a} r^{n-1} p(r \theta) d r>0
$$

is replaced by

$$
\inf _{\theta} \int_{o}^{a} r^{n-1} p(r \theta) d r>0,
$$

where $\delta$ is a positive constant.

Our next theorem depends on a lemma related to a Sobolev type embedding theorem, see [3]. It differs from such embedding results in that instead of asserting that a function belongs to a certain Lebesgue class, it states that the function minus a suitable constant is in the Lebesgue space. This lemma was communicated to the author by N. Meyers. The proof is given in an appendix.

LEMma 3.1. Let $r$ be a number with $1<r<n$ and define $r^{*}$ by the expression $\left(r^{*}\right)^{-1}=r^{-1}-n^{-1}$. Then if $u(x)$ is a function on $E^{n}$ whose gradient is in $L^{r}\left(E^{n}\right)$, there is a function $v$ in $L^{r^{*}}\left(E^{n}\right)$ and $a$ constant $k$ such that

$$
u(x)=v(x)+k .
$$

Furthermore the inequality

$$
\|v\|_{r^{*}} \leqq c\|\nabla u\|_{r}
$$

holds, where $c$ is a constant depending only on $u$ and $r$.

Now define a function $q(x)$ such that

$$
\sum_{i, j=1}^{n} a_{i j}(x) y_{i} y_{j} \geqq q(x)|y|^{2}
$$

for all $x$ and $y$ in $E^{n}$. Theorem 3.2 gives conditions on $p$ and $q$ guranteeing that $(p, a)$ has the Rellich compactness property.

TheOREM 3.2. Let $q$ be such that (3.4) holds and $\alpha$ a constant such that $(2 n)(n+2)^{-1}<\alpha \leqq 2$. Then if

(i) $p$ is in $L^{a^{*} / \alpha^{*}-2}\left(E^{n}\right)$, and

(ii) $1 / q$ is in $L^{\alpha(2-\alpha)^{-1}}\left(E^{n}\right)$

the pair $(p, a)$ has the weak Rellich compactness property.

Proof. By (3.4) we may assume without loss of generality that $a(x)=q(x) I$. Let $B$ be a bounded set in $W(p, a)$, so that there is a constant $K$ with

$$
\int p(x) u^{2} d x+\int q(x)|\nabla u|^{2} d x \leqq K
$$


for each $u$ in $B$. We must find a sequence in $B$ which is convergent in the norm for $W(p)$.

By Hölder's inequality if $\alpha<2$

$$
\begin{aligned}
\int|\nabla u|^{\alpha} d x & =\int\left[q(x)^{-1}\right]^{\alpha / 2}\left|q^{1 / 2}(x) \nabla u\right|^{\alpha} d x \\
& \leqq\left[\int\left[q(x)^{-1}\right]^{\alpha /(2-\alpha)} d x\right]^{(2-\alpha) / 2}\left[\int q(x)|\nabla u|^{2} d x\right]^{\alpha / 2} .
\end{aligned}
$$

Thus for each $u$ in $B$

$$
\int|\nabla u|^{\alpha} d x \leqq C_{1} K
$$

where $C_{1}$ depends only on $q(x)$. Clearly (3.5) also holds when $\alpha=2$. By Lemma 3.1 and (3.5) for each $u$ in $B$ there is a $v_{u}$ in $L^{\alpha^{*}}$ and a constant $k_{u}$ such that

$$
u=v_{u}+k_{u}
$$

and

$$
\left\|v_{u}\right\|_{\alpha^{*}} \leqq C_{2}
$$

where $C_{2}$ does not depend on $u$.

Now we claim there is a sequence $\left\{u_{n}\right\}$ from $B$ such that the $v_{u_{n}}$ associated with $u_{n}$ by (3.6) converge in $W(p)$. For by Hölder's inequality

$$
\int_{|x|>R} p(x) v_{u}^{2} d x \leqq\left(\int_{|x|>R}\left|v_{u}(x)\right|^{\alpha^{*}} d x\right)^{2 / \alpha^{*}}\left(\int_{|x|>R}[p(x)]^{\alpha^{*} /\left(\alpha^{*}-2\right)}\right)^{\left(\alpha^{*}-2\right) / \alpha^{*}} .
$$

So by hypothesis (i) and (3.7) the estimate

$$
\int_{|x|>R} p(x) v_{u}^{2} d x \leqq C_{2}^{2} \varepsilon(R)
$$

holds, where $\varepsilon(R) \rightarrow 0$ as $R \rightarrow \infty$. By the results of $\S 2$ and Cantor diagonalization there is a sequence $\left\{v_{u_{n}}\right\}$ which converges in the $W(p)$ norm on each compact subset of $E^{n}$. But then the estimate (3.8) show that this sequence converges globally in $W(p)$.

If $q(x)$ is not in $L^{1}\left(E^{n}\right)$, the fact that $k=0$ in (3.6) follows. Then we are done since $u_{u}=v_{u_{n}}$. But if $p(x)$ is in $L^{1}\left(E^{n}\right)$, then clearly from (3.6) and (3.8) with $R=0$ we have the inequality

$$
|k|=\text { const. }\|k\|_{W(p)} \leqq \text { const. }\left(\|u\|_{W(p)}+\left\|v_{u}\right\|_{W(p)}\right) \leqq C,
$$

where $C$ is independent of $u$ in $B$. Thus $\left\{k_{n}\right\}$ is a bounded sequence in $\boldsymbol{R}^{1}$ and must have a convergent subsequence in $\boldsymbol{R}^{1}$. But this subsequence also converges in $W(p)$. Hence $u_{n}=v_{n_{n}}+k_{u_{n}}$ has a con- 
vergent subsequence in $W(p)$. This completes the proof.

If $a(x)$ is uniformly definite then we may choose $q(x)$ to be a positive constant. Then we may take $\alpha=2$ in Theorem 3.2. As a special case of Theorem 3.2 we have the following corollary.

COROLlaRY 3.2. If $a(x)$ is uniformly definite and $p(x)$ is in $L^{n / 2}\left(E^{n}\right)$ for $n \geqq 3$, then $(p, a)$ has the weak Rellich compactness property.

In the case where $a(x)$ is uniformly definite a simple bound on the growth of $p(x)$ as $x \rightarrow \infty$ suffices to ensure a weaker compactness property of the pair $(p, \alpha)$.

THeOREM 3.3. If $a(x)$ is uniformly definite and $p(x)=o\left(|x|^{-2}\right)$ as $x \rightarrow \infty$, then the inclusion map $H(p, a) \cap L^{2}\left(E^{n}\right) \rightarrow H(p)$ is compact.

Proof. Without loss of generality assume that $a(x)=I$ and let $B$ be a bounded set in $H(p, I) \cap L^{2}\left(E^{n}\right)$. For any function in $L^{2}\left(E^{n}\right)$ with its gradient in $L^{2}\left(E^{n}\right)$ the inequality

$$
\int|x|^{2} u^{-2} d x \leqq(n-2)^{-2} \int|\nabla u|^{2} d x
$$

holds, see [4] where (3.9) is proved in greater generality. But then because of the growth condition on $p(x)$ we have the estimate

$$
\int_{|x|>R} p(x) u^{2} d x \leqq \varepsilon(R) \int|\nabla u|^{2} d x,
$$

where $\varepsilon(R) \rightarrow 0$ as $R \rightarrow \infty$. As before we pick a sequence $\left\{u_{n}\right\}$ from $B$ which converges in $H(p)$ on each compact subset of $E^{n}$. But (3.10) then shows that $\left\{u_{n}\right\}$ converges globally in $H(p)$, and the proof is complete.

The following section contains an example to show that in the case $a(x)=I$ the conditions $p(x) \in L^{n / 2}\left(E^{n}\right)$ and $p(x)=o\left(|x|^{-2}\right)$ of Corollary 3.2 and Theorem 3.2 are the best of their kind. By this is meant that $p(x) \in L^{n / 2+\varepsilon}\left(E^{n}\right)$ and $p(x)=0\left(|x|^{-2}\right)$ will not be sufficient.

4. A necessary condition. In this section we limit ourselves to the case $a(x)=I$. In this case Theorem 4.1 provides a necessary condition for $(p, I)$ to have the Rellich compactness property.

THEOREM 4.1. Suppose $(p, I)$ has the Rellich compactness property. 
If $n=2$, then $p(x)$ must be in $L^{1}\left(E^{2}\right)$. If $n>2$ then necessarily

$$
\lim _{t \rightarrow \infty} t^{2-n} \int_{|x|<t} p(x) d x=0 \text {. }
$$

Proof. For positive numbers $a$ and $k$ consider the piecewise $C^{1}$ function $u(x ; a, k)$ defined to be identically zero for $|x| \leqq a$ and $|x| \geqq a+k+1$, to be one for $|x|=a+1$, and to be linear in $|x|$ for $a \leqq|x| \leqq a+1$ and $a+1 \leqq|x| \leqq a+k+1$. If $Q[u]$ represents the quotient $\left(\int|\nabla u|^{2} d x\right)\left(\int p u^{2} d x\right)^{-1}$, then

$$
Q[u(x ; a, k)]=\frac{\text { const. }\left[(a+1)^{n}-a^{n}+k^{-2}\left((a+k+1)^{n}-(a+1)\right)\right]}{\int p u^{2} d x} .
$$

By obvious estimation of the denominator and multiplication of numerator and denominator by $k^{2-n}$ we derive the estimate

$$
\begin{aligned}
& Q[u(x ; a, k)] \\
\leqq & \frac{\text { const. }\left[k^{2-n}\left((a+1)^{n}-a^{n}\right)+\left(1+k^{-1}(a+1)\right)^{n}-\left(k^{-1}(a+1)\right)^{n}\right]}{k^{2-n} \int_{a+1<|x|<a+1+1 / 2 k} p(x) d x} .
\end{aligned}
$$

If $n=2$ and $\int p(x) d x=\infty,(4.2)$ shows that for any $a$ there is a $k$ such that $Q[u(x ; a, k)] \leqq 1$. If $n>2$ and (4.1) does not hold there is a $\delta>0$ and a sequence $\left\{t_{m}\right\}$ with $t_{m} \rightarrow \infty$ such that

$$
t_{m}^{2-n} \int_{|x|<t_{m}} p(x) d x \geqq 2 \delta
$$

for all $m$. Now if $k=k_{m} \equiv 2\left(t_{m}-a-1\right)$, the denominator of the right side of (4.2) becomes essentially

$$
\begin{aligned}
& \left(t_{m}-(a+1)\right)^{2-n}\left[\int_{0<|x|<t_{m}} p(x) d x-\int_{0<|x|<a+1} p(x) d x\right] \\
= & \left(1-\frac{a+1}{t_{m}}\right)^{2-n}\left[t_{m}^{2-n} \int_{0<|x|<t_{m}} p(x) d x-t_{m}^{2-n} \int_{0<|x|<a+1} p(x) d x\right] \\
\geqq & \delta
\end{aligned}
$$

for all large $m$. Thus in either case there is a constant $K$ such that for any given $\alpha$, there is a $k$ such that $Q[u(x ; \alpha, k)] \leqq K$.

Thus if either condition of our theorem is violated we can pick a sequence $\left\{u_{n}\right\}$ of piecewise $C^{1}$ functions with disjoint supports such that $Q\left[u_{n}\right] \leqq K$. But if we set $v_{n}=\left[\int p u_{n}^{2} d x\right]^{-1 / 2} u_{n}$, then

$$
\int p v_{n}^{2} d x+\int\left|\nabla v_{n}\right|^{2} d x=1+Q\left[u_{n}\right] \leqq 1+K
$$


for each $n$. But $\left\{v_{n}\right\}$ clearly cannot have any convergent subsequences since

$$
\int p\left(v_{n}-v_{m}\right)^{2} d x=2
$$

if $n \neq m$. Thus if either condition in our theorem is violated, the pair $(p, I)$ will not have the Rellich compactness property.

As an example consider $p(x)=|x|^{-2}$. By Theorem $4.1(p, I)$ does not have the Rellich compactness property. Yet $p(x)$ is in $L^{n / 2+\varepsilon}\left(E^{n}\right)$ for each $\varepsilon>0$ and $p(x)=0\left(|x|^{-2}\right)$ as $x \rightarrow \infty$.

5. Unconditionally nonoscillatory equations. Let $p(x)$ and $a(x)$ be as in $\S 1$ and in addition let them be $C^{\infty}$. We shall show that if $(p, a)$ has the Rellich compactness property then for each $\lambda>0$ the equation

$$
\sum_{i, j=1}^{n} \frac{\partial}{\partial x_{i}}\left(a_{i j} u_{j}\right)+\lambda p u=0
$$

is nonoscillatory. We say that in this case the equation

$$
\sum_{i, j=1}^{n} \frac{\partial}{\partial x_{i}}\left(a_{i j} u_{j}\right)+p u=0
$$

is unconditionally nonoscillatory.

THEOREM 5.1. Suppose that $(p, a)$ has either the weak or strong Rellich compactness property. Then (5.2) is unconditionally nonoscillatory.

Proof. Define $C_{0}^{\infty}(|x|>R)$ to be the space of $C^{\infty}$ functions with compact supports contained in the set of $x$ with $|x|>R$. Define the function $f(R)$ by

$$
f(R)=\inf \left\{Q[\varphi] \mid \varphi \text { is in } C_{0}^{\infty}(|x|>R)\right\},
$$

where

$$
Q[\varphi]=\left[\int \sum_{i, j=1}^{n} a_{i j}(x) \varphi_{i} \varphi_{j} d x\right]\left[\int p(x) \varphi^{2} d x\right]^{-1} .
$$

Now $f(R)$ is a continuous function of $R$ and furthermore $\lim _{R \rightarrow \infty} f(R)=$ $\infty$. For if $\lim _{R \rightarrow \infty} f(R)=L<\infty$, then we could select a sequence of functions in $C_{0}^{\infty}$, say $\left\{\varphi_{m}\right\}$, with disjoint supports such that

$$
\lim _{m \rightarrow \infty} Q\left[\varphi_{m}\right]=L \text {. }
$$


But this contradicts the fact that $(p, a)$ has the Rellich compactness property since if

$$
\psi_{m}=\left(\int p \varphi_{m} d x\right)^{-1 / 2} \varphi_{m}
$$

then

$$
\int p \psi_{m}^{2} d x+\int \sum_{i, j} \psi_{m, i} \psi_{m, j} d x=1+Q\left[\varphi_{m}\right] \leqq K
$$

for all $m$, and

$$
\int p\left(\psi_{m}-\psi_{l}\right)^{2} d x=2
$$

if $m \neq l$. So on the one hand $\left\{\psi_{m}\right\}$ forms a bounded set in $W(p, a)$ or $H(p, a)$ and on the other contains no subsequences convergent in $W(p)$ or $H(p)$.

Now if $\lambda>0$ pick $R$ so that $f(R)>\lambda$. If $N$ is any bounded smooth subdomain of $\{x|| x \mid<R\}$, the first eigenvalue $\sigma_{1}$ of the problem

$$
\begin{aligned}
& \sum_{i, j=1}^{n} \frac{\partial a}{\partial x_{0}}\left(a_{i j}(x) u_{j}\right)+\sigma p(x) u=0, \text { in } N \\
& u=0 \text { on } \partial N
\end{aligned}
$$

is greater than $f(R)$ and so $\sigma_{1}>\lambda$. Thus $N$ cannot be a nodal domain for the equation

$$
\sum_{i, j=1}^{n} \frac{\partial}{\partial x_{i}}\left(a_{i j}(x) u_{j}\right)+\lambda p(x) u=0 .
$$

So (5.4) is nonoscillatory, and (5.2) is unconditionally nonoscillatory.

CoROLlary 5.1. If $p$ and $q$ satisfy the conditions of Theorem 3.2, then (5.2) is unconditionally nonoscillatory.

COROLlaRY 5.2. If $a(x)$ is uniformly definite on $E^{n}, n \geqq 3$, either of the following conditions is sufficient for (5.2) to be unconditionally nonoscillatory:

(i) $p(x)$ is in $L^{n / 2}\left(E^{n}\right)$,

(ii) $p(x)=o\left(|x|^{-2}\right) \quad$ as $\quad|x| \rightarrow \infty$.

Condition (ii) of Corollary (5.2) is also obtained as a special case of a theorem of Headley and Swanson, see [2] Theorem 5. However, Corollary 5.1 and condition (i) of Corollary 5.2 seem to be new.

Theorem 3.1 coupled with Theorem 5.1 leads to the next corollary.

Corollary 5.3. Suppose $a(x)$ is uniformly definite on $E^{n}$ and 
$p(x)$ is positive and continuous for each $x$. If

$$
\lim _{a \rightarrow \infty}\left[\sup _{\theta} \int_{a}^{\infty} t^{n} p(r \theta) d r\right]=0
$$

then (5.2) is unconditionally nonoscillatory.

Corollary 5.3 can be used to prove a theorem of Headley, see [1], Theorem 2. If the smallest eigenvalue of $a(x)$ is greater than or equal to a positive constant $K$ and $g_{0}(r)=\max _{|x|=r} b(x)$, the equation

$$
K \sum_{i=1}^{n} \frac{\partial^{2} v}{\partial x_{i}^{2}}+g_{0}(r) v=0
$$

is a Sturmian majorant of the equation

$$
\sum_{i, j=1}^{n} \frac{\partial}{\partial x_{i}}\left(a_{i j}(x) u_{j}\right)+b(x) u=0 .
$$

If we write (5.5) as an ordinary differntial equation in $r$ and transform to remove the first derivative term, as Headley does, we arrive at the equation

$$
K y^{\prime \prime}+\left[g_{0}(r)-K(n-1)(n-3) / 4 r^{2}\right] y=0,
$$

which in turn is majorized by

$$
K y^{\prime \prime}+g_{1}^{+}(r) y=0,
$$

where $g_{1}^{+}(r)=\max \left(0, g_{0}(r)-K(n-1)(n-3) / 4 r^{2}\right)$. So if (5.7) is nonoscillatory so is (5.6). But Corollary 5.3 in the case $n=1$ shows that the condition

$$
\int_{a}^{\infty} r g_{1}^{+}(r) d r<\infty
$$

suffices. This is Headley's condition.

Appendix: Proof of Lemma 3.1. Suppose that $f_{1}, \cdots, f_{n}$ are functions in $L^{r}\left(E^{n}\right)$ with the property that $\left(\partial f_{i}\right) /\left(\partial x_{j}\right)=\left(\partial f_{j}\right) /\left(\partial x_{i}\right)$ as distributions. We shall construct a function $v$ such that

$$
\frac{\partial v}{\partial x_{i}}=f_{i}, \quad i=1, \cdots, n
$$

and

$$
\|v\|_{r^{*}} \leqq c\|f\|_{r},
$$

where $\left(r^{*}\right)^{-1}=r^{1}-n^{-1}, f=\left\{f_{1}, \cdots, f_{n}\right)$, and $c$ is a constant that 
depends only on $n$ are $r$. The proof is based on the validity of the inequality

$$
\|\varphi\|_{r^{*}} \leqq c\|\nabla \varphi\|_{r}
$$

for functions with compact support, see [3].

Let $f_{i}^{(k)}$ be a modification of $f_{i}$ so that $f_{i}^{(k)} \rightarrow f_{i}$ in $L^{r}$ as $k \rightarrow \infty$. Set $\Gamma(x)=$ const. $|x|^{2-n}$ if $n \geqq 3$ and $\Gamma(x)=$ const. $\log |x|$ if $n=2$, so that $\Delta \Gamma=\varepsilon$, where $\varepsilon$ is the Dirac distribution with support at zero, and $\Delta=\sum_{i=1}^{n} \partial^{2} /\left(\partial x_{i}^{2}\right)$. Let $\xi_{h}(x)$ be a smooth function with $0 \leqq \xi_{h} \leqq 1, \xi_{h}(x)=1$ for $|x| \leqq h, \xi_{h}(x)=0$ for $|x| \geqq 2 h,\left|\nabla \xi_{h}\right| \leqq 2 h^{-1}$. Now consider the function

$$
v_{h}^{(k)}=\sum_{i=1}^{n}\left(\xi_{h} \frac{\partial \Gamma}{\partial x_{i}}\right) *\left(f_{i}^{(k)} \xi_{h}\right) .
$$

Differentiate and use the fact that $\left(\partial f_{i}\right) /\left(\partial x_{j}\right)=\left(\partial f_{j}\right) /\left(\partial x_{i}\right)$, so that

$$
\begin{aligned}
& \frac{\partial v_{h}^{(k)}}{\partial x_{j}}=\sum_{i=1}^{n}\left(\xi_{h} \frac{\partial \Gamma}{\partial x_{i}}\right) *\left(\frac{\partial f_{j}^{(k)}}{\partial x_{i}} \xi_{h}\right)+\sum_{i=1}^{n}\left(\xi_{h} \frac{\partial \Gamma}{\partial x_{i}}\right) *\left(\frac{\partial \xi_{h}}{\partial x_{j}} f_{i}^{(k)}\right) \\
= & \sum_{i=1}^{n} \frac{\partial}{\partial x_{i}}\left(\xi_{h} \frac{\partial \Gamma}{\partial x_{i}}\right) *\left(f_{j}^{(k)} \xi_{h}\right)+\sum_{i=1}^{n} \xi_{h} \frac{\partial \Gamma}{\partial x_{i}} *\left(\frac{\partial \xi_{h}}{\partial x_{j}} f_{i}^{(k)}-f_{j}^{(k)} \frac{\partial \xi_{h}}{\partial x_{j}}\right) .
\end{aligned}
$$

Finally

$$
\text { (A.4) } \begin{aligned}
& \frac{\partial v_{h}^{(k)}}{\partial x_{j}} \\
= & f_{j}^{(k)} \xi_{h}+\sum_{i=1}^{n}\left(\frac{\partial \xi_{h}}{\partial x_{i}} \frac{\partial \Gamma}{\partial x_{i}}\right) *\left(f_{j}^{(k)} \xi_{h}\right)+\sum_{i=1}^{n} \xi_{h} \frac{\partial \Gamma}{\partial x_{i}} *\left(\frac{\partial \xi_{h}}{\partial x_{j}} f_{i}^{(k)}-f_{j}^{(k)} \frac{\partial \xi_{h}}{\partial x_{j}}\right) .
\end{aligned}
$$

For each $i$

$$
\left\|\frac{\partial \xi_{h}}{\partial x_{i}} \frac{\partial \Gamma}{\partial x_{i}} * f_{j}^{(k)} \xi_{h}\right\|_{r} \leqq\left\|f_{j}^{(k)}\right\|_{r}\left\|\frac{\partial \xi_{h}}{\partial x_{i}} \frac{\partial \Gamma}{\partial x_{i}}\right\|_{1} \leqq \text { const }\|f\|_{r},
$$

so that the first summation term on the right in (A.4) is bounded in $L^{r}$ uniformly in $h$. Furthermore we find that for each $i$ the same term is tending to zero in $L_{10 c}^{r}$ as $h \rightarrow 0$. For if $B_{a}$ is the set of $x$ with $|x| \leqq a$, Hölder's inequality implies that

$$
\begin{aligned}
\int_{B_{a}}\left|\frac{\partial \xi_{h}}{\partial x_{i}} \frac{\partial \Gamma}{\partial x_{i}} * f_{j}^{(k)} \xi_{h}\right|^{r} d x & \leqq \text { const. } \int\left|f_{j}^{(k)}(x)\right|^{r} \int_{B_{0}}\left|\frac{\partial \xi_{h}}{\partial x_{i}} \frac{\partial \Gamma}{\partial x_{i}}(y-x)\right| d y d x \\
& \leqq \text { const. } \int_{|x| \geq h-a}\left|f_{j}(x)\right|^{r} d x \longrightarrow 0 \text { as } h \longrightarrow \infty
\end{aligned}
$$

Furthermore Hölder's inequality also shows that all the other terms in the right side of (A.4) tend to zero in $L^{r}$ as $h \rightarrow \infty$.

So $\left(\partial v_{h}^{(k)}\right) /\left(\partial x_{j}\right)-f_{j}^{(k)}$ is bounded in $L^{r}$ as $h \rightarrow \infty$ and converges to 
zero in $L_{10 c}^{r}$. For some sequence of $h$ 's then tending to infinity,

$$
\frac{\partial v_{h}^{(k)}}{\partial x_{j}} \longrightarrow f_{j}^{(k)}
$$

weakly in $L^{r}$. Also (A.3) holds for each $v_{h}^{(k)}$ so there is a further subsequence of $h$ 's with $v_{h}^{(k)} \rightarrow v^{(k)}$ weakly in $L^{r^{*}}$. Hence $\left(\partial v^{(k)}\right) /\left(\partial x_{j}\right)=$ $f_{j}^{(k)}$ as distributions and (A.2) holds for $v=v^{(k)}$ and $f=f^{(k)}$. Finally for a subsequence of $k$ 's tending to $\infty, v^{(k)} \rightarrow v$ weakly in $L^{r^{*}}$ and $\left(\partial v^{(k)}\right) /\left(\partial x_{j}\right) \rightarrow f_{j}$ in $L^{r}$. So $(\partial v) /\left(\partial x_{j}\right)=f_{j}$ and (A.2) holds. This completes the proof.

\section{REFERENCES}

1. V. B., Headley, Some oscillation properties of selfadjoint elliptic equations, Proc. Amer. Math., Soc., 25 (1970), 824-829.

2. V. B. Headley and C. A. Swanson, Oscillation criteria for elliptic equations, Pacific J. Math., 27 (1968), 501-506.

3. L. Nirenberg, On elliptic partial differential equations, Ann. della Scuola Nor. Sup. Pisa, 13 (1959), 115-162.

4. John Piepenbrink, Integral inequalities and theorems of Liouville type, J. Math. Anal. Appl., 26 (1969), 630-639.

5. S. L. Sobolev, On a theorem of functional analysis, Amer. Math. Soc. Translations, (2) 34 (1963), 39-68.

6. - Einige Anwendungen der Funktionalanalysis auf Gleichungen der Mathematischen Physic, Akademie-Verlag, Berlin 1964.

7. G. Stampachia and M. K. V. Murthy, Boundary value problems for some degenerateelliptic operators, Ann. di Mat. pura ed. Appl. LXXX, (1968) 1-122.

Received June 24, 1971. This work was in part supported by NSF grant GP-7041X. and Air Force Grant AFOSR 883-67.

UNIVERSITY OF MinNESOTA 


\section{PACIFIC JOURNAL OF MATHEMATICS}

\section{EDITORS}

H. SAMELSON

Stanford University

Stanford, California 94305

C. R. HobBY

University of Washington

Seattle, Washington 98105
J. DUGUNDJI

Department of Mathematics

University of Southern California

Los Angeles, California 90007

RICHARD ARENS

University of California

Los Angeles, California 90024

\section{ASSOCIATE EDITORS}

E. F. BECKENBACH

B. H. NeumanN

F WoLF

K. YoshidA

\section{SUPPORTING INSTITUTIONS}

UNIVERSITY OF BRITISH COLUMBIA

UNIVERSITY OF SOUTHERN CALIFORNIA

CALIFORNIA INSTITUTE OF TECHNOLOGY

UNIVERSITY OF CALIFORNIA

MONTANA STATE UNIVERSITY

STANFORD UNIVERSITY

UNIVERSITY OF NEVADA

NEW MEXICO STATE UNIVERSITY

OREGON STATE UNIVERSITY

UNIVERSITY OF OREGON

OSAKA UNIVERSITY

UNIVERSITY OF TOKYO

UNIVERSITY OF UTAH

WASHINGTON STATE UNIVERSITY

UNIVERSITY OF WASHINGTON

$\stackrel{*}{*} \stackrel{*}{*} \stackrel{*}{*}{ }^{*}{ }^{*}$ MMEICAN MATHEMATICAL SOCIETY

NAVAL WEAPONS CENTER

Printed in Japan by International Academic Printing Co., Ltd., Tokyo, Japan 


\section{Pacific Journal of Mathematics}

\section{Vol. 42, No. $1 \quad$ January, 1972}

Tage Bai Andersen, On Banach space valued extensions from split faces ........

David Marion Arnold, A duality for quotient divisible abelian groups of finite

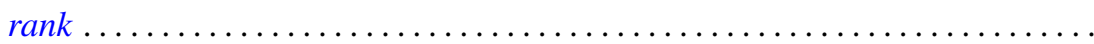

Donald Pollard Ballou, Shock sets for first order nonlinear hyperbolic

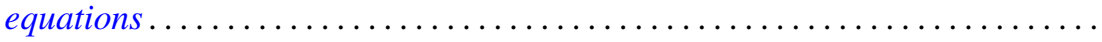

Leon Brown and Lowell J. Hansen, On the range sets of $H^{p}$ functions .........

Alexander Munro Davie and Arne Stray, Interpolation sets for analytic

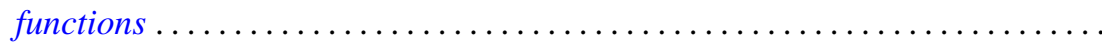

M. G. Deshpande, Structure of right subdirectly irreducible rings. II . . . . . . . . .

Barry J. Gardner, Some closure properties for torsion classes of abelian

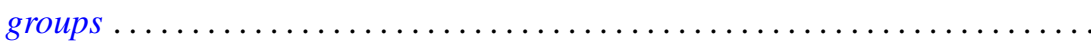

Paul Daniel Hill, Primary groups whose subgroups of smaller cardinality are

direct sums of cyclic groups . . . . . . . . . . . . . . . . . . .

Richard Allan Holzsager, When certain natural maps are equivalences .........

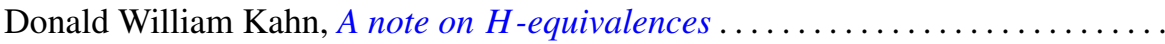

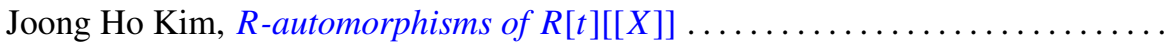

Shin'ichi Kinoshita, On elementary ideals of polyhedra in the 3-sphere.........

Andrew T. Kitchen, Watts cohomology and separability...

Vadim Komkov, A technique for the detection of oscillation of second order

ordinary differential equations .

Charles Philip Lanski and Susan Montgomery, Lie structure of prime rings of characteristic 2

Andrew Lenard, Some remarks on large Toeplitz determinants . .

Kathleen B. Levitz, A characterization of general Z.P.I.-rings. II .

Donald A. Lutz, On the reduction of rank of linear differential systems

David G. Mead, Determinantal ideals, identities, and the Wronskian ...

Arunava Mukherjea, A remark on Tonelli's theorem on integration in product

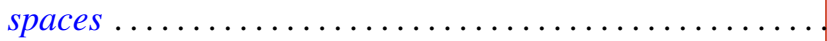

Hyo Chul Myung, A generalization of the prime radical in nonassociative rings.

John Piepenbrink, Rellich densities and an application to unconditionally nonoscillatory elliptic equations.

Michael J. Powers, Lefschetz fixed point theorems for a new class of multi-valued maps .

Aribindi Satyanarayan Rao, On the absolute matrix summability of a Fourier

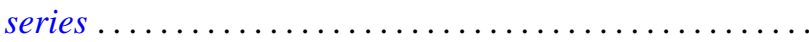

T. S. Ravisankar, On Malcev algebras ......................... 227

William Henry Ruckle, Topologies on sequences spaces . . . . . . . . . . . . . 235

Robert C. Shock, Polynomial rings over finite dimensional rings . . . . . . . . . 251

Richard Tangeman, Strong heredity in radical classes . . . . . . . . . . . . . . 259

B. R. Wenner, Finite-dimensional properties of infinite-dimensional spaces . . . . 267 